\title{
Relation between three-qubit entanglement invariants and two-qubit concurrence
}

\author{
Wen-Long Yang ${ }^{1}$ and Jing-Ling Chen ${ }^{1, *}$ \\ ${ }^{1}$ Theoretical Physics Division, Chern Institute of Mathematics, Nankai University, Tianjin 300071, P.R.China
}

\begin{abstract}
In this Brief Report we show the relation between three-qubit entanglement invariants and twoqubit concurrence with the help of projective measurements. How to use these invariants to represent the entanglement property of three-qubit pure states is also investigated.
\end{abstract}

PACS numbers: 03.65.Ud, 03.67.Mn

Quantum entanglement and quantum nonlocality are regarded as a key feature of quantum mechanics since its early days [1, 2]. Recently, by taking advantage of the entanglement property there has emerged an attractive research field called quantum information, which is composed of quantum cryptography, quantum teleportation, quantum computation and so on [3]. Frequent use of entanglement has imminently called for a precise and proper description of entanglement for quantum states. For two-qubit state, entanglement entropy and entanglement of formation and the well-known concurrence [4, 5, 6, 7, 8, 9] are all good measures for the degree of entanglement for two-qubit states. During the period of finding a quantity as a measure of the degree of entanglement for three-qubit states, five local-unitary-transformation invariants have been thought to be important resources to form such a quantity [10, 11, 12, 13, 14, 15]. Efforts have also been devoted to find such a quantity to measure the entanglement of three-qubit pure states from the viewpoint Bell inequality [16]. However, three qubits can be entangled in two inequivalent ways, namely the Greenberger-Horne-Zeilinger (GHZ) state [17] and the $W$ state [18], therefore finding only one unified quantity to describe the entanglement of all three-qubit states seems to be not easy.

In 2002, Linden et al. pointed out that almost every pure state of three qubits is completely determined by its two-particle reduced density matrices [19]. This may hint that there is a definite relation between five localunitary-transformation entanglement invariants of threequbit between two-qubit concurrence. To our knowledge, such a relation has not been reported in the literature before. The purpose of this Brief Report is to show the exact relation between the three-qubit entanglement invariants and the two-qubit concurrence with the help of the projective measurements.

The report is organized as follows. First, we show the relation between local-unitary-transformation invariants of three-qubit pure states and concurrence of two-qubit pure states via projective measurements. Then, we investigate how to use these invariants to represent the entanglement property of three-qubit pure states. Conclusion is made in the end.

*Electronic address: chenjl@nankai.edu.cn
A two-qubit state is the spin state or pseudo-spin state composed of two spin- $1 / 2$ particles $A$ and $B$, which reads

$$
\left|\psi_{2}\right\rangle=\mu_{00}|00|+\mu_{01}|01\rangle+\mu_{10}|10\rangle+\mu_{11}|11\rangle .
$$

The state is separable means that it can be written in the following direct-product form

$$
\left(a|0\rangle_{A}+b|1\rangle_{A}\right) \otimes\left(c|0\rangle_{B}+d|1\rangle_{B}\right),
$$

otherwise it is entangled or inseparable. Concurrence is one of proper measures of the degree of entanglement for two-qubit states. It is first defined in [8] to find a simple way to quantify the entanglement of two-qubit mixed state $\rho_{A B}$

$$
C=\max \left\{\lambda_{1}-\lambda_{2}-\lambda_{3}-\lambda_{4}, 0\right\},
$$

where $\lambda_{i}$ is the square root of eigenvalues of $\rho_{A B} \cdot\left(\sigma_{y} \otimes\right.$ $\left.\sigma_{y}\right) \cdot \rho_{A B}^{*} \cdot\left(\sigma_{y} \otimes \sigma_{y}\right)$ in decreasing order. The definition is also work for pure-state case of two qubits, in this case it possesses a simpler form

$$
C=2 \sqrt{\operatorname{det}\left(\rho_{A}\right)}=2 \sqrt{\operatorname{det}\left(\rho_{B}\right)},
$$

where $\rho_{A}$ and $\rho_{B}$ are density matrices obtained from the pure state $\rho_{A B}$ by tracing out the other particle. The concurrence (4) is a local-unitary-transformation invariant for $\rho_{A B}$ which means $C\left(\rho_{A B}\right)=C\left(\left(U_{A} \otimes\right.\right.$ $\left.\left.U_{B}\right) \rho_{A B}\left(U_{A} \otimes U_{B}\right)^{\dagger}\right)$. For two-qubit pure state (11), the concurrence is usually expressed in terms of the coefficients $\mu_{i j}$ as

$$
C=2\left|\mu_{00} \mu_{11}-\mu_{01} \mu_{10}\right| \text {. }
$$

$C$ is 0 for separable state (2) and 1 for maximally entangled state $(1 / \sqrt{2})(|00\rangle+|11\rangle)$.

Local-unitary-transformation invariants for threequbit states have been found for about one decade 10, 11, 12, 13, 14]. For a three-qubit pure state

$$
|\psi\rangle=\sum_{i, j, k=0,1} \mu_{i j k}|i j k\rangle,
$$

the indices $i, j$ and $k$ stand for the spin states of three spin-1/2 particles $A, B$ and $C$. More precisely, the five linear independent entanglement invariants (i.e., $I_{1}$ to $I_{5}$ ) are [14, 20]

$$
I_{0}=\langle\psi \mid \psi\rangle
$$




$$
\begin{aligned}
I_{1} & =\operatorname{Tr}\left(\rho_{A}^{2}\right), \\
I_{2} & =\operatorname{Tr}\left(\rho_{B}^{2}\right) \\
I_{3} & =\operatorname{Tr}\left(\rho_{C}^{2}\right) \\
I_{4} & =3 \operatorname{Tr}\left(\rho_{A} \otimes \rho_{B} \cdot \rho_{A B}\right)-\operatorname{Tr}\left(\rho_{A}^{3}\right)-\operatorname{Tr}\left(\rho_{B}^{3}\right), \\
I_{5} & =16 \mid \mu_{000}^{2} \mu_{111}^{2}+\mu_{001}^{2} \mu_{110}^{2}+\mu_{010}^{2} \mu_{101}^{2}+\mu_{011}^{2} \mu_{100}^{2} \\
& -2\left(\mu_{000} \mu_{111} \mu_{011} \mu_{100}+\mu_{000} \mu_{111} \mu_{101} \mu_{010}\right. \\
& +\mu_{000} \mu_{111} \mu_{110} \mu_{001}+\mu_{011} \mu_{100} \mu_{101} \mu_{010} \\
& \left.+\mu_{011} \mu_{100} \mu_{110} \mu_{001}+\mu 101 \mu_{010} \mu_{110} \mu_{001}\right) \\
& +\left.4\left(\mu_{000} \mu_{011} \mu_{101} \mu_{110}+\mu_{111} \mu_{001} \mu_{010} \mu_{100}\right)\right|^{2}
\end{aligned}
$$

where $I_{0}$ is the identity and a homogeneous polynomial of degree two. $I_{1}, I_{2}$ and $I_{3}$ are homogeneous polynomials of degree four, $I_{4}$ is a homogeneous polynomial of degree six and $I_{5}$ is a homogeneous polynomial of degree eight.

Though there are several generalizations of two-qubit concurrence to multi-particle concurrence [21, 22], the relation between (5) and (7) has not yet been revealed before. In the following we would like to show an intimate relation between them.

We first make a projective measurement $\left|\psi_{A}\right\rangle\left\langle\psi_{A}\right|$ with respect to particle $A$ with

$$
\left|\psi_{A}\right\rangle=\cos \frac{\theta_{A}}{2}|0\rangle_{A}+\sin \frac{\theta_{A}}{2} e^{i \phi_{A}}|1\rangle_{A} .
$$

This is like measuring the spin of $A$ in the direction $\left(\sin \theta_{A} \cos \phi_{A}, \sin \theta_{A} \sin \phi_{A}, \cos \theta_{A}\right)$. After performing the measurement, one gets the collapsed state as

$$
\left|\psi_{B C}\right\rangle=\frac{\left\langle\psi_{A} \mid \psi\right\rangle}{\sqrt{W_{B C}\left(\theta_{A}, \phi_{A}\right)}},
$$

where

$$
W_{B C}\left(\theta_{A}, \phi_{A}\right)=\left\langle\psi \mid \psi_{A}\right\rangle\left\langle\psi_{A} \mid \psi\right\rangle \text {. }
$$

Namely, after this measurement, one gets a pure twoqubit state $\left|\psi_{B C}\right\rangle$ with the probability $W_{B C}\left(\theta_{A}, \phi_{A}\right)$. We can calculate the concurrences $C_{B C}\left(\theta_{A}, \phi_{A}\right)$ of $\left|\psi_{B C}\right\rangle$ with the definition (5). $C_{B C}\left(\theta_{A}, \phi_{A}\right)$ is an invariant under local unitary transformation of $B$ and $C$, which is the property of the concurrence. From (10), one may show that $W_{B C}\left(\theta_{A}, \phi_{A}\right)$ is also an invariant under local unitary transformation of $B$ and $C$. The local unitary transformation of $A$ rotates $\left|\psi_{A}\right\rangle$ in its spin space, we found the integration of $W_{B C}^{2}\left(\theta_{A}, \phi_{A}\right) C_{B C}^{2}\left(\theta_{A}, \phi_{A}\right)$ in the whole spin space of $A$ is invariant under local unitary transformations of $A, B$ and $C$. Now we denote this quantity as

$$
\begin{aligned}
C_{B C 4}= & \int_{0}^{\pi} d \theta_{A} \int_{0}^{2 \pi} d \phi_{A} \sin \theta_{A} \\
& W_{B C}^{2}\left(\theta_{A}, \phi_{A}\right) C_{B C}^{2}\left(\theta_{A}, \phi_{A}\right),
\end{aligned}
$$

where the squared concurrence $C_{B C}^{2}\left(\theta_{A}, \phi_{A}\right)$ has been adopted, such that the integrand does not contain the square-root calculation and the integration becomes easier to perform. Similarly, we can get $C_{A B 4}$ and $C_{A C 4}$.
Here we have added a 4 in the subscript to denote that $C_{A B 4}, C_{A C 4}$ and $C_{B C 4}$ are all homogeneous polynomial invariants of degree four. Such an approach can be generalized to obtain polynomials of higher degree just by raising the order of the weight $W_{B C}\left(\theta_{A}, \phi_{A}\right)$. For instances, the polynomial invariants of degree six can be obtained through

$$
\begin{aligned}
C_{B C 6}= & \int_{0}^{\pi} d \theta_{A} \int_{0}^{2 \pi} d \phi_{A} \sin \theta_{A} \\
& W_{B C}^{3}\left(\theta_{A}, \phi_{A}\right) C_{B C}^{2}\left(\theta_{A}, \phi_{A}\right),
\end{aligned}
$$

and the polynomial invariants of degree eight can be achieved by

$$
\begin{aligned}
C_{B C 8}= & \int_{0}^{\pi} d \theta_{A} \int_{0}^{2 \pi} d \phi_{A} \sin \theta_{A} \\
& W_{B C}^{4}\left(\theta_{A}, \phi_{A}\right) C_{B C}^{4}\left(\theta_{A}, \phi_{A}\right) .
\end{aligned}
$$

$C_{A B 6}, C_{A C 6}, C_{A B 8}$ and $C_{A C 8}$ can be obtained in the same way. They are all homogeneous polynomials which are invariant under local unitary transformation, therefore they should be able to be expressed by the set of polynomial invariants $I$ 's mentioned in Eq. (7). After making some observations, we found their relations as

$$
\begin{aligned}
C_{A B 4} & =\frac{\pi}{3}\left(-I_{1}-I_{2}+I_{3}+I_{0}^{2}\right), \\
C_{A C 4} & =\frac{\pi}{3}\left(-I_{1}+I_{2}-I_{3}+I_{0}^{2}\right), \\
C_{B C 4} & =\frac{\pi}{3}\left(I_{1}-I_{2}-I_{3}+I_{0}^{2}\right), \\
C_{A B 6} & =\frac{\pi}{18}\left[2 I_{0}^{3}-3 I_{0}\left(I_{1}+I_{2}-2 I_{3}\right)-2 I_{4}\right], \\
C_{A C 6} & =\frac{\pi}{18}\left[2 I_{0}^{3}-3 I_{0}\left(I_{1}-2 I_{2}+I_{3}\right)-2 I_{4}\right], \\
C_{B C 6} & =\frac{\pi}{18}\left[2 I_{0}^{3}-3 I_{0}\left(-2 I_{1}+I_{2}+I_{3}\right)-2 I_{4}\right], \\
C_{A B 8} & =\frac{\pi}{240}\left[12\left(-I_{1}-I_{2}+I_{3}+I_{0}^{2}\right)^{2}-I_{5}\right] \\
C_{A C 8} & =\frac{\pi}{240}\left[12\left(-I_{1}+I_{2}-I_{3}+I_{0}^{2}\right)^{2}-I_{5}\right] \\
C_{B C 8} & =\frac{\pi}{240}\left[12\left(I_{1}-I_{2}-I_{3}+I_{0}^{2}\right)^{2}-I_{5}\right] .
\end{aligned}
$$

It is not hard to obtain the I's from the $C$ 's, i.e.,

$$
\begin{aligned}
I_{1} & =I_{0}^{2}-\frac{3}{2 \pi}\left(C_{A B 4}+C_{A C 4}\right), \\
I_{2} & =I_{0}^{2}-\frac{3}{2 \pi}\left(C_{A B 4}+C_{B C 4}\right), \\
I_{3} & =I_{0}^{2}-\frac{3}{2 \pi}\left(C_{A C 4}+C_{B C 4}\right), \\
I_{4} & =I_{0}^{3}-\frac{9}{4 \pi}\left[4 C_{A B 6}+I_{0}\left(-2 C_{A B 4}+C_{A C 4}+C_{B C 4}\right)\right] \\
& =I_{0}^{3}-\frac{9}{4 \pi}\left[4 C_{A C 6}+I_{0}\left(C_{A B 4}-2 C_{A C 4}+C_{B C 4}\right)\right] \\
& =I_{0}^{3}-\frac{9}{4 \pi}\left[4 C_{B C 6}+I_{0}\left(C_{A B 4}+C_{A C 4}-2 C_{B C 4}\right)\right],
\end{aligned}
$$




$$
\begin{aligned}
I_{5} & =\frac{12}{\pi}\left[\pi\left(-I_{1}-I_{2}+I_{3}+I_{0}^{2}\right)^{2}-20 C_{A B 8}\right] \\
& =\frac{12}{\pi}\left[\pi\left(-I_{1}+I_{2}-I_{3}+I_{0}^{2}\right)^{2}-20 C_{A C 8}\right] \\
& =\frac{12}{\pi}\left[\pi\left(I_{1}-I_{2}-I_{3}+I_{0}^{2}\right)^{2}-20 C_{B C 8}\right] .
\end{aligned}
$$

This is the relation between three-qubit entanglement invariants and two-qubit concurrence via projective measurements. It is worthy to mention that there is another way to obtain the polynomial invariants of degree eight:

$$
\begin{aligned}
C_{B C 8}^{\prime}= & \int_{0}^{\pi} d \theta_{A} \int_{0}^{2 \pi} d \phi_{A} \sin \theta_{A} \\
& W_{B C}^{4}\left(\theta_{A}, \phi_{A}\right) C_{B C}^{2}\left(\theta_{A}, \phi_{A}\right) .
\end{aligned}
$$

and also $C_{A B 8}^{\prime}$ and $C_{A C 8}^{\prime}$. They can also be expressed by the $I$ 's as

$$
\begin{aligned}
C_{A B 8}^{\prime}= & \frac{\pi}{480}\left[I_{5}-64 I_{4} I_{0}+4\left(-I_{1}^{2}-I_{2}^{2}+7 I_{3}^{2}\right)\right. \\
& +8\left(-3 I_{1}-3 I_{2}+17 I_{3}\right) I_{0}^{2} \\
& \left.+8\left(I_{1} I_{2}-3 I_{1} I_{3}-I_{2} I_{3}\right)-4 I_{0}^{4}\right] \\
C_{A C 8}^{\prime}= & \frac{\pi}{480}\left[I_{5}-64 I_{4} I_{0}+4\left(-I_{1}^{2}+7 I_{2}^{2}-I_{3}^{2}\right)\right. \\
& +8\left(-3 I_{1}+17 I_{2}-3 I_{3}\right) I_{0}^{2} \\
& \left.+8\left(-3 I_{1} I_{2}+I_{1} I_{3}-3 I_{2} I_{3}\right)-4 I_{0}^{4}\right] \\
C_{B C 8}^{\prime}= & \frac{\pi}{480}\left[I_{5}-64 I_{4} I_{0}+4\left(7 I_{1}^{2}-I_{2}^{2}-I_{3}^{2}\right)\right. \\
& +8\left(-3 I_{1}+17 I_{2}-3 I_{3}\right) I_{0}^{2} \\
& \left.+8\left(-3 I_{1} I_{2}+I_{1} I_{3}-3 I_{2} I_{3}\right)-4 I_{0}^{4}\right] .
\end{aligned}
$$

The different is that these formula are more complicated than the previous ones as in Eq. (14).

From the definitions (11), (12) and (13), we can measure these $C$ 's. One notices that two-qubit concurrence for pure state can be obtained locally from (4) by measuring the density matrix of either party of the two-qubit state. Such a discussion has been made in [23] and a recent experiment 24] has been carried out to realize it. Moreover the probability, e. g. $W_{B C}\left(\theta_{A}, \phi_{A}\right)$, can also be obtained locally. Therefore for an unknown three-qubit pure state shared by Alice, Bob and Charlie, $C_{B C}\left(\theta_{A}, \phi_{A}\right)$ is locally measured by Bob and $W_{B C}\left(\theta_{A}, \phi_{A}\right)$ is locally measured by Alice. After Alice scanned all $\theta_{A}$ and $\phi_{A}$, and they tell each other the quantity they obtained through classical channel, then they can compute $C_{B C 4}, C_{B C 6}$ and $C_{B C 8}$ with (11), (12) and (13). By changing their roles in turn, they will know all the invariants of the state, or say, they know all entanglement properties of the state. So theoretically, we can get the entanglement property of the pure entangled threequbit state by local measurement and classical communication.

Now we investigate how to use these five local-unitarytransformation invariants (7) to represent the entanglement properties of three-qubit pure states. For two-qubit system, we know that the concurrence, as a local-unitarytransformation invariant, is a good measure of degree of

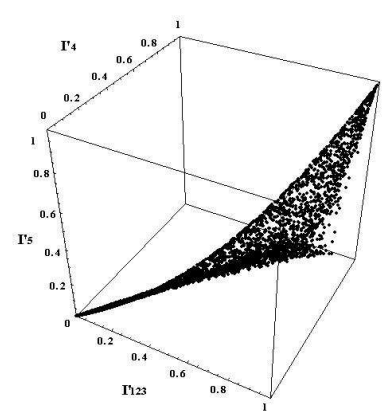

FIG. 1: 5000 points for random three-qubit states are plotted in the $I_{123}^{\prime}-I_{4}^{\prime}-I_{5}^{\prime}$ coordinate system.

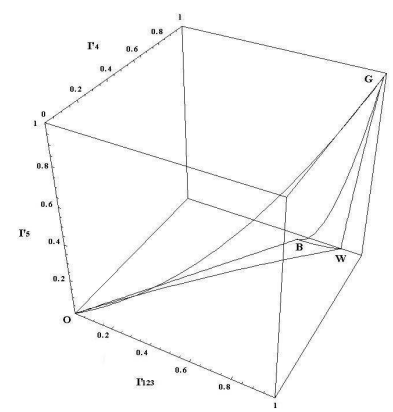

FIG. 2: This figure shows the line boundary of all pure threequbit states in the $I_{123}^{\prime}-I_{4}^{\prime}-I_{5}^{\prime}$ coordinate system. The point $O$ stands for the states like $|\psi\rangle_{O}$. The point $B$ stands for the states like $1 / \sqrt{2}|000\rangle+1 / \sqrt{2}|011\rangle$, in which one of the three particles is not entangled with the other two maximally entangled particles. $W$ stands for the states like $|\psi\rangle_{W}$, and $G$ stands for the states like $|\psi\rangle_{G}$.

entanglement. But three-qubit system has two kinds of different entanglement, i.e., the GHZ class and the $W$ class [18], thus it might be more appropriate that we use more than one quantity to represent the entanglement property of three qubits. Luckily, we have five localunitary-transformation invariants. It can be seen from (77) that $I_{1}, I_{2}$ and $I_{3}$ are not invariant under the permutation of the three particles and $I_{4}$ and $I_{5}$ are invariant under such a permutation. In order to eliminate the difference brought by the permutations between particles, we use $I_{123}=I_{1}+I_{2}+I_{3}, I_{4}$ and $I_{5}$ as our measurements for the entanglement of three-qubit pure states. In such a coordinate system, when we talk about a point for a state, this point corresponds to states which can be transformed to the state by local unitary transformations and together with permutations of $A, B$ and $C$. We find that $I_{123}$ has its maximum value 3 for the direct-product states $|\psi\rangle_{O}=|000\rangle$, and its minimum value 1.5 for the GHZ state, i.e., $|\psi\rangle_{G}=1 / \sqrt{2}(|000\rangle+|111\rangle) ; I_{4}$ reaches its maximum value 1 for $|\psi\rangle_{O}$ and its minimum value $2 / 9$ for the $W$ state, i.e., $1 / \sqrt{3}\left(|001\rangle+|010\rangle+|100\rangle\right.$; and $I_{5}$ ranges from 1 for $|\psi\rangle_{G}$ to 0 for $|\psi\rangle_{W}$ and $|\psi\rangle_{O}$. To make the figure looks better, we rescale three new quantities 
as

$$
\begin{aligned}
I_{123}^{\prime} & =2\left(3-I_{123}\right) / 3 \\
I_{4}^{\prime} & =9\left(1-I_{4}\right) / 7, \quad I_{5}^{\prime}=I_{5},
\end{aligned}
$$

which are all range from 0 to 1 . An arbitrary three-qubit state has its corresponding position in the coordinate system $I_{123}^{\prime}-I_{4}^{\prime}-I_{5}^{\prime}$. The origin stands for the direct-product state $|\psi\rangle_{O} ;|\psi\rangle_{G}$ has maximum 1 in the axes $I_{123}^{\prime}$ and $I_{5}^{\prime}$, and $|\psi\rangle_{W}$ has maximum 1 in the axis $I_{4}^{\prime}$. $I_{4}^{\prime}$ and $I_{5}^{\prime}$ could be the two quantities describing two distinct three-body entanglements [10, 18]. To make it visible, points for 5000 random three-qubit states have been plotted in Fig. [1] in $I_{123}^{\prime}-I_{4}^{\prime}-I_{5}^{\prime}$ coordinate system.

We also find the line boundary of Fig. 1, which is shown in Fig. 2, It is of interest to show the corresponding states in the line boundary. In Fig. 2. the line $O G$ stands for generalized $G H Z$ states $\cos \theta|000\rangle+\sin \theta|000\rangle$ with $\theta \in[0, \pi / 2] . O B$ are states $\cos \theta|000\rangle+\sin \theta|011\rangle$ with $\theta \in[0, \pi / 2]$. $O W$ are states like $(\cos \theta / \sqrt{3})(|011\rangle+|101\rangle+|110\rangle)+\sin \theta|111\rangle$ with $\theta \in[0, \pi / 2]$. $B W$ are states $(\cos \theta / \sqrt{2})(|000\rangle+|111\rangle)+$ $(\cos \theta / \sqrt{2})(|100\rangle+|011\rangle)$ with $\theta \in[0, \pi] . \quad B G$ are states $(\sin \theta / \sqrt{2})(|101\rangle+|000\rangle)+\cos \theta|110\rangle$ with $\theta \in[\arctan \sqrt{2}, \pi / 2]$. And $W G$ are states $(\sin \theta / \sqrt{6})(|001\rangle+|010\rangle+|100\rangle+|011\rangle+|101\rangle+|110\rangle)+$ $(\cos \theta / \sqrt{2})(|000\rangle+|111\rangle)$, with $\theta \in[2 / 3 \pi, 5 \pi / 6]$. These state are lie in the line boundary of the figure, it might be because they are the nearest ways (or more precisely orbits [6]) between four states $|\psi\rangle_{O},|\psi\rangle_{B},|\psi\rangle_{W}$ and $|\psi\rangle_{G}$ in the $S U(8)$ parameter space of three qubits.

Let us see how the points in Fig. 1 represent the entanglement properties of three-qubit states. We know that all two-body entangled states are on $O B$. Ref. [19] indicated that the generalized $W$ states $|\psi\rangle_{W}^{\prime}=$ $a|001\rangle+b|010\rangle+c|100\rangle$ were uniquely determined by their two-party reduced states. We can understand this from Fig. 2 by calculating their $I_{5}^{\prime}$, and we know that all $|\psi\rangle_{W}^{\prime}$ have zero $I_{5}^{\prime}$. Though we cannot say that the distance from a point to the origin in the $I_{123}^{\prime}-I_{4}^{\prime}-I_{5}^{\prime}$ coordinate system can measure the degree of entanglement of the three-qubit state, at least we can get a brief impression of the entanglement property of the state. The point on or near $O B$ will be more likely a two-particle entangled state, and a state very near the origin should be less entangled than those who are much far from the origin.

In conclusion, we show the relation between threequbit entanglement invariants and two-qubit concurrence with the help of projective measurements. This relation also provides a method to get three-qubit localunitary-transformation invariants by local measurement and classical communication. These five invariants can form three new quantities (18), which are invariant under both local unitary transformation and permutations. In the $I_{123}^{\prime}-I_{4}^{\prime}-I_{5}^{\prime}$ coordinate, we can distinguish two-body entanglement state and the generalized $W$ states from other states. For a three-qubit state, it is helpful for us to know some of its entanglement property by plotting its corresponding point in the $I_{123}^{\prime}-I_{4}^{\prime}-I_{5}^{\prime}$ coordinate system. It is also interesting and significant to apply this approach to multi-qubit systems, which we shall investigate subsequently.

ACKNOWLEDGMENTS This work is supported from NSF of China (Grant No. 10605013) and Program for New Century Excellent Talents in University.
[1] A. Einstein, B. Podolsky, and N. Rosen, Phys. Rev. 47, 777 (1935).

[2] J. S. Bell, Physics (N.Y.) 1, 195 (1964).

[3] M.A. Nielsen and I. L. Chuang, Quantum Computation and Quantum Information (Cambridge University Press, Cambridge, England, 2000).

[4] C. H. Bennett, H. J. Bernstein, S. Popescu, and B. Schumacher, Phys. Rev. A 53, 2046 (1996).

[5] C. H. Bennett, D. P. DiVincenzo, J. A. Smolin, and W. K. Wootters, Phys. Rev. A 54, 3824 (1996).

[6] S. Popescu, and D. Rohrlich, Phys. Rev. A 56, R3319 (1997).

[7] V. Vedral, M. B. Plenio, M. A. Rippin, and P. L. Knight, Phys. Rev. Lett. 78, 2275 (1997).

[8] S. Hill, and W. K. Wootters, Phys. Rev. Lett. 78, 5022 (1997)

[9] W. Wootters, Phys. Rev. Lett. A 80, 2245 (1998).

[10] J. Schlienz, and G. Mahler, Phys. Lett. A 224, 39 (1996).

[11] N. Linden, and S. Popescu, Fortschr. Phys. 46, 4-5, 567578 (1998).

[12] M. Grassl, M. Rötteler, and T. Beth, Phys. Rev. A 58, 1833 (1998).

[13] J. Kempe, Phys. Rev. A 60, 910 (1999).
[14] A. Sudbery, J. Phys. A 34, 643 (2000).

[15] P. Lévay, Phys. Rev. A 71, 012334 (2005).

[16] J. L. Chen, C. F. Wu, L. C. Kwek, and C. H. Oh, Phys. Rev. Lett. 93, 140407 (2004).

[17] D. M. Greenberger, M. A. Horne, and A. Zeilinger, in Bells Theorem, Quantum Theory, and Conceptions of the Universe, edited by M. Kafatos (Kluwer, Dordrecht, 1989), p. 73.

[18] W. Dür, G. Vidal, and J. I. Cirac, Phys. Rev. A 62, 062314 (2000).

[19] N. Linden, S. Popescu, and W. K. Wootters, Phys. Rev. Lett. 89, 207901 (2002).

[20] V. Coffman, J. Kundu, and W. K. Wootters, Phys. Rev. A 61, 052306 (2000).

[21] F. Mintert, M. Kuś, and A. Buchleitner, Phys. Rev. Lett. 95, 260502 (2005).

[22] H. Heydari, J. Phys. A 39, 15225 (2006).

[23] J. M. G. Sancho, and S. F. Huelga, Phys. Rev. A 61, 042303 (2000).

[24] S. P. Walborn, P. H. Souto Ribeiro, L. Davidovich, F. Mintert, and A. Buchleitner, Nature 440, 1022 (2006). 